\title{
FIRST RESULTS OF CONTINUOUS IUE OBSERVATIONS OF ALGOL
}

\author{
R. González-Riestra \\ ILE Observatory \\ II LSSPA \\ P. O. Box 50727 \\ 28080 Madrid \\ spain
}

\author{
A. Giménez \\ LAEFF \\ IIISPA \\ P. O. Box 50727 \\ 28080 Madrid \\ Spain
}

\author{
E.F. Guinan \\ Dpt. of Astronomy and Astrophrsics \\ Villanova University \\ Villanova \\ PA 1908.5 \\ ISSA
}

ABSTRACT. We present the first results of a continuous monitoring of Algol with IUE along 1.5 orbital period (4 1/3 days). A total of 32 high resolution spectra were obtained. Algol represents the final slow stage of mass transfer. The spectra show the presence of high ionization lines (NV, SiIV, CIV) due to the heating of the gas that impacts the accreting star. The depth of the primary eclipse is larger at shorter wavelengths and the shape of the continuum out of the eclipse is compatible with a B star, ruling out the presence of an extra light source at these wavelengths.

Ultraviolet observations have been of great importance in the study of Algol systems. They have confirmed the generally accepted model of the gas flow, showing the existence of a non-thermal energy source, as evidenced by the presence of high ionization absorption lines and allowing the study of the gas turbulences. Since these systems have substantial long term variations, it is important not to mix observations taken in different orbital cycles. In September 1989 we carried out continuous observations of three Algol systems with IUE over at least one full orbit (see details of the program in Gimenez et al. 1990). We present here the first results on Algol.

Algol itself is the brightest prototype of this class of semi-detached binaries. It is a triple system (an eclipsing pair and an A star invisible in the ultraviolet). The eclipsing binary consist of a B8 $V$ primary and a KO-2 secondary. The physical and orbital properties of Algol are well determined (see Table 1). A schematic picture of the most probable configuration of Algol is shown in Guinan (1989). The small mass ratio of the system indicates that it is in the final, slow stage of mass transfer and mass loss. This terminal stage appears to be the most common and Jongest for Algol-type binaries. 
$\underline{\text { Table } 1}$

Properties of the system

\begin{tabular}{|c|c|c|}
\hline & $\begin{array}{l}\text { Period } \\
\text { e } \\
\text { i } \\
\text { a } \\
\text { q } \\
\text { distance }\end{array}$ & $\begin{array}{l}2.48673 \\
0.0 \\
81^{\circ} .50 \\
14 \text { Ro } \\
0.220 \\
25 \text { pc }\end{array}$ \\
\hline & Hot star (gainer) & Cool star (loser) \\
\hline $\begin{array}{l}\text { Spectral type } \\
\text { Temperature } \\
\text { Radius } \\
\text { Mass }\end{array}$ & $\begin{array}{l}\mathrm{B} 8 \mathrm{~V} \\
12500 \mathrm{~K} \\
2.90 \mathrm{Ro} \\
3.70 \mathrm{Mo}\end{array}$ & $\begin{array}{ll}\mathrm{KO}-2 & \mathrm{IV} \\
5100 \mathrm{~K} \\
3.50 & \mathrm{Ro}_{\mathrm{o}} \\
0.82 \mathrm{Mo}\end{array}$ \\
\hline
\end{tabular}

\section{Table 2}

\section{Continuum bands}

\begin{tabular}{ll}
\hline $1284.0-86.0$ & $2094.0-95.0$ \\
$1380.0-82.0$ & $2308.0-10.0$ \\
$1450.5-52.5$ & $2407.0-09.0$ \\
$1538.0-40.0$ & $2508.0-10.0$ \\
$1659.0-61.0$ & $2619.0-20.0$ \\
$1764.5-66.5$ & $2713.0-14.0$ \\
$1849.0-51.0$ & $2809.0-11.0$ \\
$1949.0-51.0$ & $2919.0-21.0$ \\
$2034.0-34.0$ & $2999.0-3000.0$ \\
\hline
\end{tabular}

Ultraviolet continuum light
curves were constructed in
regions of the spectra tree of
strong features one or two A
wide, and roughly separated by
lud A. The selected bands are
listed in Table 2. lhey are
generally quite well behaver,
as shown in Figure 1. They are
tound to be also similar to the
FEs (Fine Frror sensor) light
curve obtainedduring the same
observing run (Figure 2 ). Only
a small decrease of around $10 \%$
in brightness near phase 0.9 can
be noticed. This is at
the same phase when absorption
lines reach maximum st.rength.

Ultraviolet continuum light curves were constructed in regions of the spectra free of features one or two A luv A. The selected bands are listed in Table 2. They are generally quite well behaver, as shown in Figure 1. They are tound to be also similar to the FES (Fine Frror Sensor) light curve obtainedduring the same observing run (Figure 2). Only a small decrease of around $10 \%$ in brightness near phase 0.9 can the same phase when absorption lines reach maximum strength.

The strong enhancement of the lines at that phase is due to the visibility of the impact site of gas from the cool star on the inner, trailing hemisphere of the B8 V star (see scheme in Guinan, 1990). 


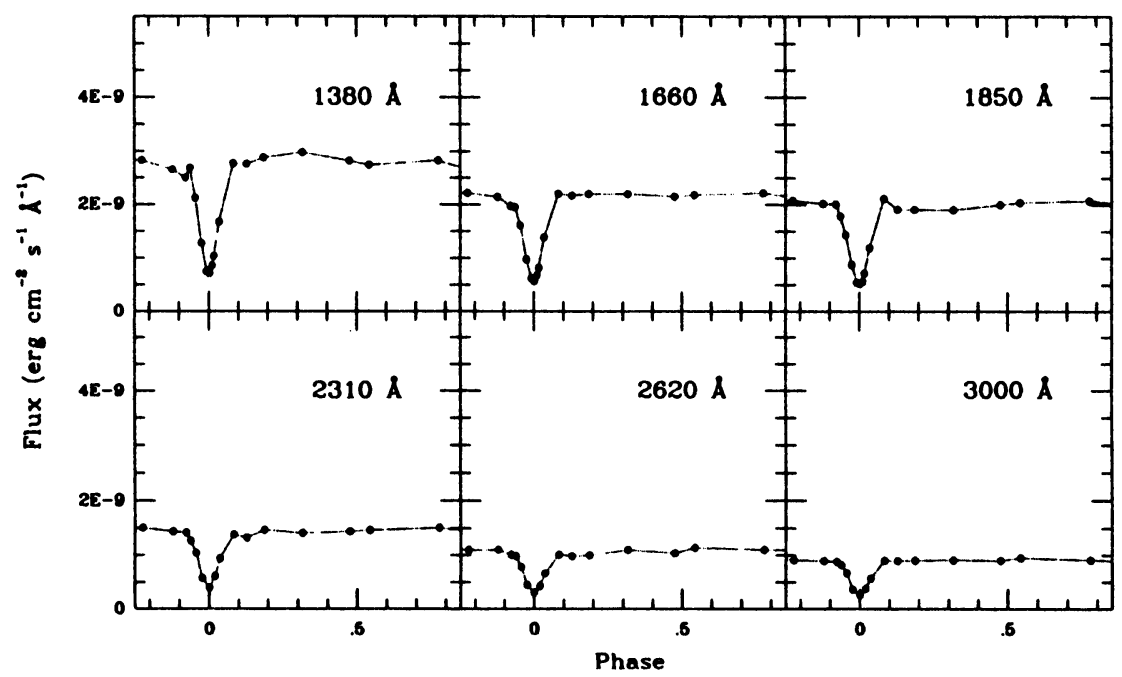

Figure 1. Examples of ultraviolet light curves of Algol at several wavelengths

As expected, the primary eclipse is deeper and the secondary is nearly invisible in the ultraviolet. The change of depth in the primary eclipse, when the cool secondary eclipses the brighter primary, is shown in Figure 3, where we have also included the values from the Johnson's $B$ and $V$ filters as derived from the observations by Kim (1989).

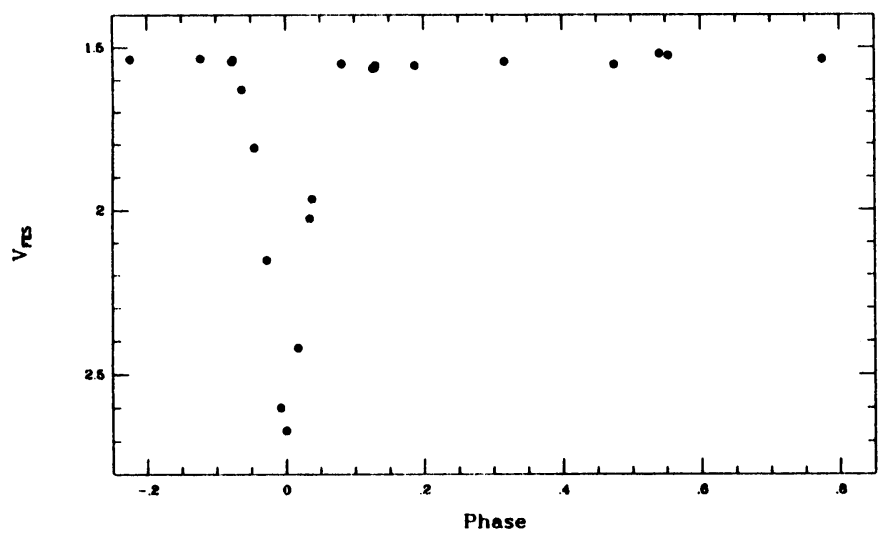

Figure 2. Fine Errror Sensor light curve of Algol obtained during the IUF observations. The FES has an effective wavelength of $5200 \mathrm{~A}$, and the photometric: accuracy of these measurements is 0.015 magnitudes. 
Finally, Figure 1 shows the flux distribution outside ot eclipse, taken at phase 0.32. Ohatrvations for the $B$ and $f$ filters (him. 1989) aris also plottrod.

\section{REFERENCES}

Gimènez, A., González-Riestra, R., Guinan, E.f., hondo, Y., McCluskey, G.E., Bradstreet, D.H., McCook, G.P., Dorren, J.ll., Johansson, S., Sahade, l. (1990) in "Evolution in Astrophysics", ESA SP-310, p. 383

Guinan, E.F. (1989) Sp. Sci. Ker. 50, 35

(juinan, E.F. (1990) in "Evolution in Astrophysics", ESA SP-310, p. 73

him. H.I. (1989) Ap. J. 342, 1061

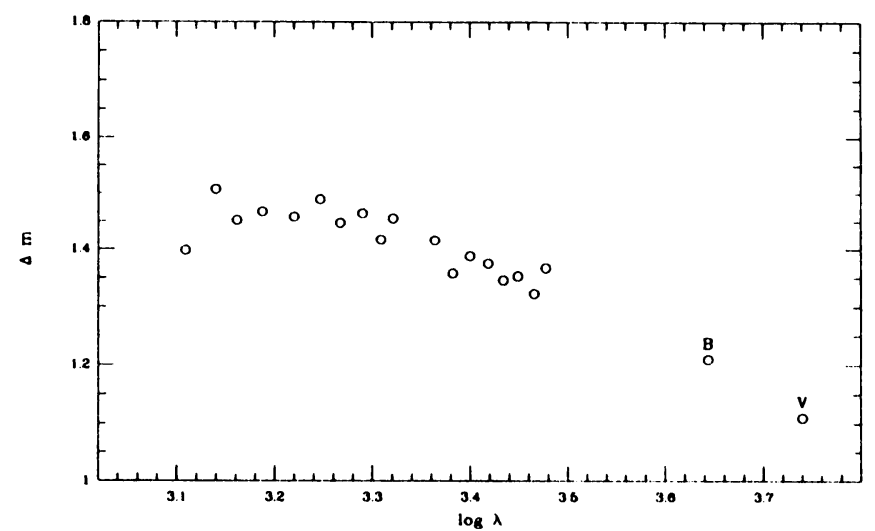

Figure 3. Variation of the depth of the primary eclipse with wavelength.

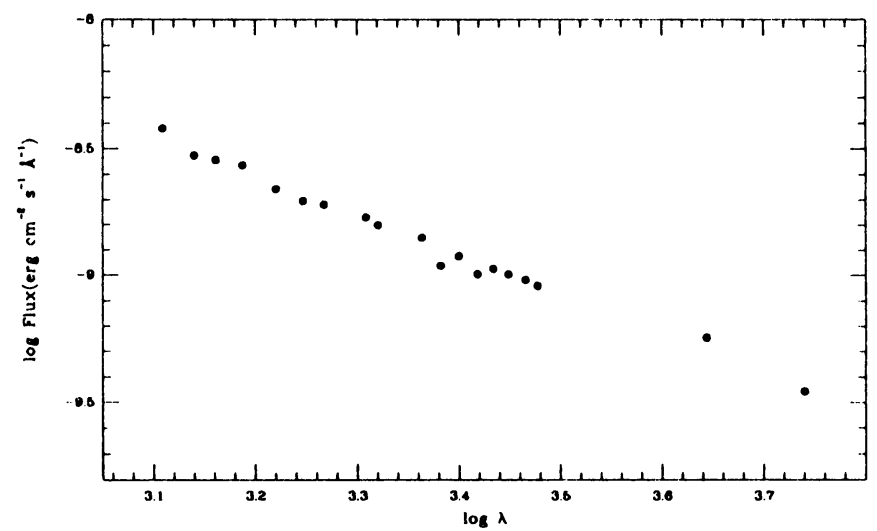

Figure 4. Ultraviolet to Visible out-of-eclipse spectral distribution of Algol. 